\section{Endoscopic ultrasound diagnosis of a small splenic vein aneurysm}
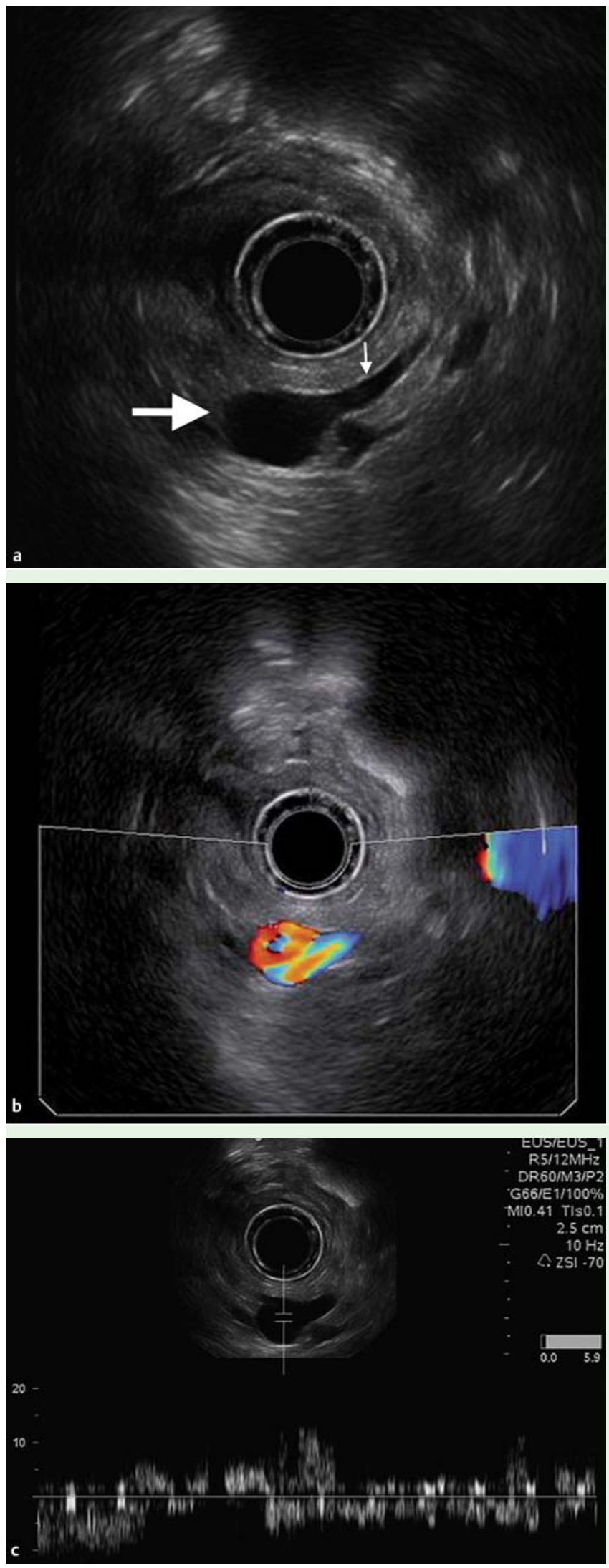

Fig. 1 Endoscopic ultrasound (EUS) images of a splenic vein aneurysm using: a B-mode, which shows a localized dilatation of the splenic vein (large arrow) and the intact part of the splenic vein (small arrow); b color Doppler, which shows the blood flow in the Doppler, which shows continuous venous flow. lesion; c pulsed-wave
Splenic vein aneurysm (SVA) is an extremely rare vascular abnormality. Although endoscopic ultrasound (EUS) can detect vascular structures, the characteristic findings of an SVA have not been reported. We describe a patient with an SVA found incidentally on an EUS that was performed for gastric antral ectopic pancreas. The diagnosis was based on the findings during EUS using an electronic radial scanning echoendoscope (EG-530UR; Fujifilm Corp., Saitama, Japan) with color Doppler flow-mapping capabilities.

We identified a focally dilated distal splenic vein at the level of the pancreatic tail ( $\bullet$ Fig. 1 a, b), and a venous flow signal was detected by pulsed-wave Doppler ultrasound ( $\bullet$ Fig. 1c). Based on these findings, we diagnosed an SVA. Subsequent venous-phase three-dimensional computed tomography angiography (CTA) revealed a venous aneurysm on the splenic vein, confirming the final diagnosis of SVA ( $\bullet$ Fig. 2).

A splenic artery aneurysm (SAA) is similar to an SVA in form and shape, and must be distinguished from an SVA. We previously reported the characteristic EUS findings of SAAs [1]. EUS using pulsed-wave Doppler mode shows a pulsating arterial wave in an SAA. In contrast, it shows continuous flow in an SVA. Therefore, we believe that the typical EUS findings of an SVA are focal dilatation of the splenic vein in B-mode images with continuous flow in the pulsed-wave Doppler mode.

The best treatment strategy for incidentally detected asymptomatic SVAs remains controversial $[2,3]$. We elected to monitor this lesion rather than perform surgery, and there was no change on either abdominal ultrasound or EUS at the 48-month follow-up. To our knowledge, this is the first reported case of an SVA diagnosed by EUS.

Endoscopy_UCTN_Code_CCL_1AF_2AG_3AD

Competing interests: None

Keishi Komori ${ }^{1}$, Kazuya Akahoshi', Yasuaki Motomura', Masaru Kubokawa', Junya Gibo ${ }^{1}$, Kenji Kanayama', Shinichiro Fukuda ${ }^{1}$, Shohei Hamada ${ }^{1}$, Yoshiaki Otuka ${ }^{1}$, Kazuhiko Nakamura ${ }^{2}$

${ }^{1}$ Department of Gastroenterology, Aso lizuka Hospital, lizuka, Japan

2 Department of Medicine and Bioregulatory Science, Graduate School of Medical Sciences, Kyushu University, Fukuoka, Japan 


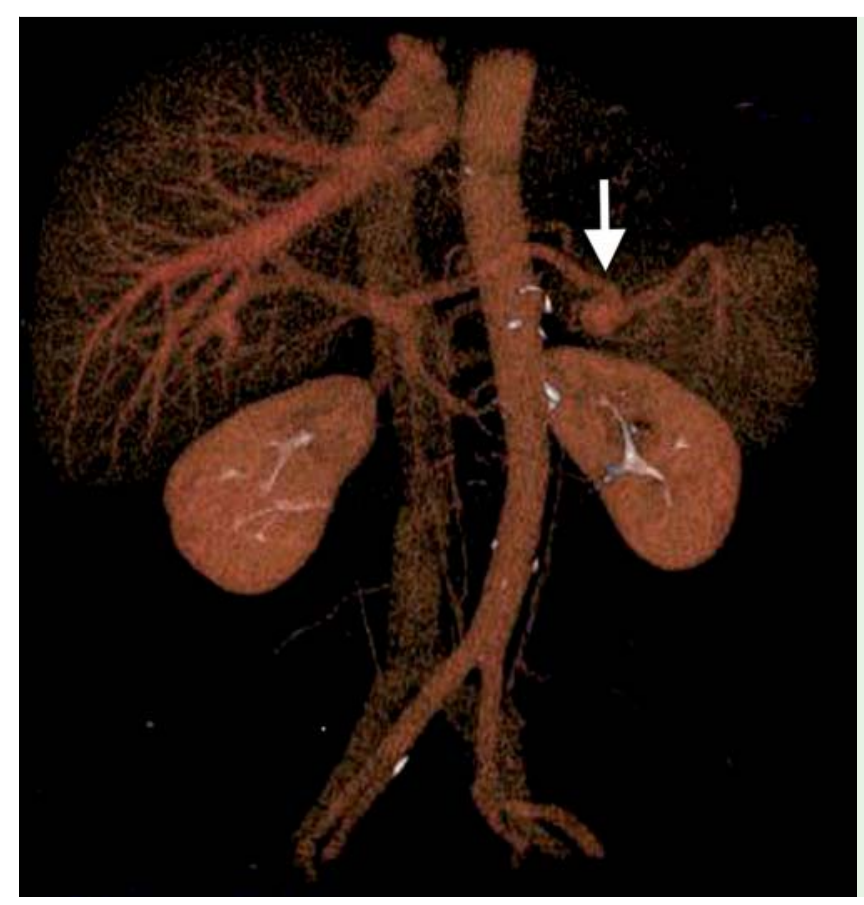

Fig. 2 Three-dimensional computed tomography angiography showing an aneurysm ( $17 \mathrm{~mm}$ in diameter) at the hilum of the splenic vein.

\section{References}

1 Higuchi N, Akahoshi K, Honda Ket al. Diagnosis of a small splenic artery aneurysm mimicking a gastric submucosal tumor on endoscopic ultrasound. Endoscopy 2010; 42 (Suppl. 02): E107-E108

2 Comert M, Erdem LO, Ozdolap S et al. Splenic vein aneurysm demonstrated by magnetic resonance angiography. Dig Dis Sci 2005; 50: $1344-1346$

3 Torres G, Hines GL, Monteleone F et al. Splenic vein aneurysm: is it a surgical indication? J Vasc Surg 1999; 29: 719-721

\section{Bibliography}

DOI http://dx.doi.org/

10.1055/s-0033-1358930

Endoscopy 2014; 46: E5-E6

(c) Georg Thieme Verlag KG

Stuttgart · New York

ISSN 0013-726X

\section{Corresponding author}

\section{Kazuya Akahoshi, MD, PhD}

Department of Gastroenterology

Aso lizuka Hospital

3-83 Yoshio

lizuka 820-8505

Japan

Fax: +81-948-298747

kakahoshi2@aol.com 